\title{
Squeezed Fock state by inconclusive photon subtraction
}

\author{
Stefano Olivares $\ddagger$ and Matteo G. A. Paris $\S$ \\ Dipartimento di Fisica, Università degli Studi di Milano, Italia
}

\begin{abstract}
We analyze in details the properties of the conditional state recently obtained by J. Wenger et al. [Phys. Rev. Lett. 92, 153601 (2004)] by means of inconclusive photon subtraction (IPS) on a squeezed vacuum state $S(r)|0\rangle$. The IPS process can be characterized by two parameters: the IPS transmissivity $\tau$ and the photodetection quantum efficiency $\eta$. We found that the conditional state approaches the squeezed Fock state $S(r)|1\rangle$ when $\tau, \eta \rightarrow 1$, i.e., in the limit of singlephoton subtraction. For non-unit IPS transmissivity and efficiency, the conditioned state remains close to the target state, i.e. shows a high fidelity for a wide range of experimental parameters. The nonclassicality of the conditional state is also investigated and a nonclassicality threshold on the IPS parameters is derived.
\end{abstract}

\section{Introduction}

Beam splitters (BS) and avalanche photodetectors (APDs) play a fundamental role in quantum information processing. These key elements, among the other applications, can be used in order to generate non-Gaussian states from Gaussian ones [1, 2, 3, 4, and to distill continuous-variable entanglement [5].

In this paper we focus our attention on the output state recently obtained experimentally by J. Wenger et al. 6] by means of photon subtraction on a squeezed vacuum state $S(r)|0\rangle, S(r)$ being the squeezing operator. More precisely, when a Gaussian state, such as $S(r)|0\rangle$, is mixed with the vacuum at a beam splitter and, then, on/off photodetection is performed on the reflected beam, an unknown number of photons is subtracted from the input state and the output state is no longer Gaussian, i.e., the input state is de-Gaussified: this is due to the fact that the positive operator valued measure (POVM) describing the APD is non-Gaussian. Since the actual number of detected photons cannot be resolved by the APD, in [3] we referred to this process as to inconclusive photon subtraction (IPS). In general the IPS process can be characterized by two parameters: the beam splitter transmissivity $\tau$ and the quantum efficiency $\eta$ of the APD. As we will see, the conditional output state obtained by IPS on a squeezed vacuum is close to the squeezed Fock state $S(r)|1\rangle$, which is otherwise difficult to produce

$\ddagger$ Stefano.Olivares@mi.infn.it

$\S$ Matteo.Paris@fisica.unimi.it 


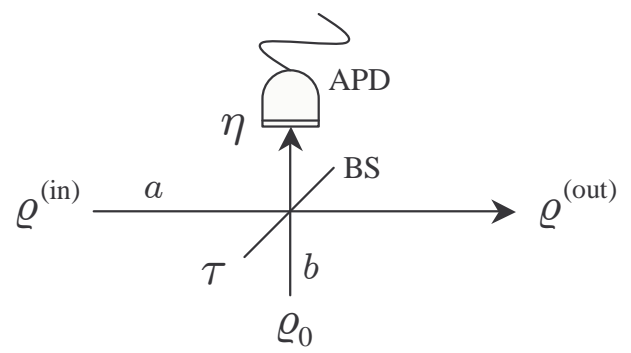

Figure 1. Scheme of the IPS process: the input state $\varrho^{(\text {in) }}$ is mixed with the vacuum state $\varrho_{0}=|0\rangle\langle 0|$ at a beam splitter (BS) with transmissivity $\tau$; then, avalanche photodetection (APD) with quantum efficiency $\eta$ is performed on the reflected beam. When the detector clicks we obtain the IPS state $\varrho^{\text {(out) }}$.

by Hamiltonian processes. For this reason, we address IPS as an effective resource to generate those squeezed Fock states. We find that the IPS conditional state reduces to $S(r)|1\rangle$ in the limit $\tau, \eta \rightarrow 1$, whereas for different values of the transmissivity and of the quantum efficiency it remains close to this target state, showing a high fidelity for a wide range of the parameters. Finally, since the IPS state obtained from the squeezed vacuum is, in general, non classical and mixed, we study how the purity and the nonclassical depth of the IPS state depend on $\tau, \eta$, and on the input squeezing parameter $r$.

The paper is structured as follows: in Section 2 we review the main elements of the IPS process on a single mode of radiation. The fidelity between the IPS conditional state and the squeezed Fock state $S(z)|1\rangle$, as well as its purity are then investigated in Section 3. whereas section 4 is devoted to the analysis of the nonclassicality of the IPS state. Finally, Section 5 closes the paper with some concluding remarks.

\section{The inconclusive photon subtraction process}

The scheme of the inconclusive photon subtraction (IPS) process is sketched in figure 1. An input state $\varrho^{(\text {in) }}$ is mixed with the vacuum state $\varrho_{0}=|0\rangle\langle 0|$ at a beam splitter (BS) with transmissivity $\tau$ and, then, on/off avalanche photodetection (APD) with quantum efficiency $\eta$ is performed on the reflected beam. Since the APD con only distinguish the presence from the absence of light, this measurement is inconclusive, namely does not resolve the number of the detected photons. In this way, when the detector clicks, an unknown number of photon is subtracted from the initial state and we obtain the IPS state $\varrho^{\text {(out) }}$. Since the whole process is characterized by $\tau$ and $\eta$, we will refer to them also as IPS transmissivity and IPS quantum efficiency.

If the input state of the mode $a$ is the squeezed vacuum state $\varrho_{r}^{(\mathrm{in})}=|0, r\rangle\langle 0, r|$, where $|0, r\rangle=S(r)|0\rangle, S(r)=\exp \left\{\frac{1}{2} r\left(a^{\dagger^{2}}-a^{2}\right)\right\}$ being the squeezing operator (for the sake of the simplicity, without lack of generality, we can assume $r$ as real), its (Gaussian) characteristic function $\chi_{r}^{(\mathrm{in})}\left(\boldsymbol{\Lambda}_{a}\right) \equiv \chi\left[\varrho_{r}^{(\mathrm{in})}\right]\left(\boldsymbol{\Lambda}_{a}\right)$ reads

$$
\chi_{r}^{(\mathrm{in})}\left(\boldsymbol{\Lambda}_{a}\right)=\exp \left\{-\frac{1}{2} \boldsymbol{\Lambda}_{a}^{T} \boldsymbol{\sigma}_{r} \boldsymbol{\Lambda}_{a}\right\}
$$


Squeezed Fock state by inconclusive photon subtraction

where $\boldsymbol{\Lambda}=\left(\mathrm{x}_{a}, \mathrm{y}_{a}\right)^{T},(\cdots)^{T}$ being the transposition operation, and

$$
\boldsymbol{\sigma}_{r}=\frac{1}{2}\left(\begin{array}{cc}
\cosh r+\sinh r & 0 \\
0 & \cosh r-\sinh r
\end{array}\right),
$$

is the covariance matrix. Analogously, the vacuum state $\varrho_{0}=|0\rangle\langle 0|$ of the mode $b$ is described by the (Gaussian) characteristic function

$$
\chi_{0}\left(\boldsymbol{\Lambda}_{b}\right) \equiv \chi\left[\varrho_{0}\right]\left(\boldsymbol{\Lambda}_{b}\right)=\exp \left\{-\frac{1}{2} \boldsymbol{\Lambda}_{b}^{T} \boldsymbol{\sigma}_{0} \boldsymbol{\Lambda}_{b}\right\},
$$

where $\boldsymbol{\sigma}_{0}=\frac{1}{2} \mathbb{1}_{2}, \mathbb{1}_{2}$ being the $2 \times 2$ identity matrix. Since the initial two-mode state $\varrho_{r}^{(\text {in) }} \otimes \varrho_{0}$ is Gaussian, under the action of the BS its $4 \times 4$ covariance matrix

$$
\boldsymbol{\sigma}_{\text {in }}=\left(\begin{array}{c|c}
\boldsymbol{\sigma}_{r} & \mathbf{0} \\
\hline \mathbf{0} & \boldsymbol{\sigma}_{0}
\end{array}\right)
$$

transforms as follows 7

$$
\boldsymbol{\sigma}_{\mathrm{in}} \rightsquigarrow \boldsymbol{\sigma}^{\prime} \equiv \boldsymbol{S}_{\mathrm{BS}}^{T} \boldsymbol{\sigma}_{\mathrm{in}} \boldsymbol{S}_{\mathrm{BS}} \equiv\left(\begin{array}{c|c}
\boldsymbol{A} & \boldsymbol{C} \\
\hline \boldsymbol{C}^{T} & \boldsymbol{B}
\end{array}\right),
$$

where $\boldsymbol{A}, \boldsymbol{B}$, and $\boldsymbol{C}$ are $2 \times 2$ matrices and

$$
\boldsymbol{S}_{\mathrm{BS}}=\left(\begin{array}{c|c}
\sqrt{\tau} \mathbb{1}_{2} & \sqrt{1-\tau} \mathbb{1}_{2} \\
\hline-\sqrt{1-\tau} \mathbb{1}_{2} & \sqrt{\tau} \mathbb{1}_{2}
\end{array}\right),
$$

is the symplectic transformation associated to the evolution operator of the BS. Now, the on/off photodetector with quantum efficiency $\eta$ can be described by the POVM $\left\{\Pi_{\text {off }}(\eta), \Pi_{\text {on }}(\eta)\right\}$, with

$$
\Pi_{\mathrm{off}}(\eta)=\sum_{k=0}^{\infty}(1-\eta)^{k}|k\rangle\langle k|, \quad \Pi_{\mathrm{on}}(\eta)=\mathbb{I}-\Pi_{\mathrm{off}}(\eta),
$$

which corresponds to the characteristic functions

$$
\begin{aligned}
& \chi\left[\Pi_{\mathrm{off}}(\eta)\right](\boldsymbol{\Lambda}) \equiv \chi_{\eta}^{(\mathrm{off})}(\boldsymbol{\Lambda})=\frac{1}{\eta} \exp \left\{-\frac{1}{2} \boldsymbol{\Lambda}^{T} \boldsymbol{\sigma}_{\mathrm{M}} \boldsymbol{\Lambda}\right\}, \\
& \chi\left[\Pi_{\mathrm{on}}(\eta)\right](\boldsymbol{\Lambda}) \equiv \chi_{\eta}^{(\mathrm{on})}(\boldsymbol{\Lambda})=2 \pi \delta^{(2)}(\boldsymbol{\Lambda})-\chi_{\eta}^{(\mathrm{off})}(\boldsymbol{\Lambda}),
\end{aligned}
$$

respectively, $\delta^{(2)}(\boldsymbol{\Lambda})$ being the 2-dim Dirac's delta function, and

$$
\boldsymbol{\sigma}_{\mathrm{M}}=\frac{2-\eta}{2 \eta} \mathbb{1}_{2}
$$

The probability of a click in the detector is then given by $[7$

$$
\begin{aligned}
p_{\text {on }}(r, \tau, \eta) & =\operatorname{Tr}_{a b}\left[\varrho_{r, \tau}^{\prime} \mathbb{I} \otimes \Pi_{\text {on }}(\eta)\right] \\
& =\frac{1}{(2 \pi)^{2}} \int_{\mathbb{R}^{4}} d^{2} \boldsymbol{\Lambda}_{a} d^{2} \boldsymbol{\Lambda}_{b} \chi\left[\varrho_{r, \tau}^{\prime}\right]\left(\boldsymbol{\Lambda}_{a}, \boldsymbol{\Lambda}_{b}\right) \chi[\mathbb{I}]\left(-\boldsymbol{\Lambda}_{a}\right) \chi_{\eta}^{(\mathrm{on})}\left(-\boldsymbol{\Lambda}_{b}\right) \\
& =1-\left(\eta \sqrt{\operatorname{Det}\left[\boldsymbol{B}+\boldsymbol{\sigma}_{\mathrm{M}}\right]}\right)^{-1}=1-\left(\sqrt{1+\left(1-\tau_{\text {eff }}^{2}\right) \sinh ^{2} r}\right)^{-1},
\end{aligned}
$$

where $\chi\left[\varrho_{r, \tau}^{\prime}\right]\left(\boldsymbol{\Lambda}_{a}, \boldsymbol{\Lambda}_{b}\right)$ is the two-mode characteristic function associated to the state $\varrho_{r, \tau}^{\prime} \equiv U_{\mathrm{BS}} \varrho_{r}^{\text {(in) }} \otimes \varrho_{0} U_{\mathrm{BS}}^{\dagger}, \chi[\mathbb{I}](\boldsymbol{\Lambda})=2 \pi \delta^{(2)}(\boldsymbol{\Lambda})$, and $\tau_{\text {eff }} \equiv \tau_{\text {eff }}(\tau, \eta)=1-\eta(1-\tau)$. Note 
Squeezed Fock state by inconclusive photon subtraction

that when $\tau_{\text {eff }} \rightarrow 1$, the probability (13) can be approximated at the first order in $\tau_{\text {eff }}$ as follows

$$
p_{\text {on }}(r, \tau, \eta)=\left(1-\tau_{\text {eff }}\right) \sinh ^{2} r+o\left[\left(1-\tau_{\text {eff }}\right)^{2}\right] .
$$

Finally, the output state

$$
\varrho_{r, \tau, \eta}^{(\text {out })}=\frac{\operatorname{Tr}_{b}\left[\varrho_{r, \tau}^{\prime} \mathbb{I} \otimes \Pi_{\text {on }}(\eta)\right]}{p_{\text {on }}(r, \tau, \eta)},
$$

conditioned to a click of the on/off photodetector, has the following characteristic function $\chi_{r, \tau, \eta}^{\text {(out) }}\left(\boldsymbol{\Lambda}_{a}\right) \equiv \chi\left[\varrho_{r, \tau, \eta}^{\text {(out })}\right]\left(\boldsymbol{\Lambda}_{a}\right)$ :

$$
\begin{aligned}
\chi_{r, \tau, \eta}^{\text {(out })}\left(\boldsymbol{\Lambda}_{a}\right) & =\frac{1}{2 \pi p_{\text {on }}(r, \tau, \eta)} \int_{\mathbb{R}^{2}} d^{2} \boldsymbol{\Lambda}_{b} \chi\left[\varrho_{r, \tau}^{\prime}\right]\left(\boldsymbol{\Lambda}_{a}, \boldsymbol{\Lambda}_{b}\right) \chi_{\eta}^{\text {(on })}\left(-\boldsymbol{\Lambda}_{b}\right) \\
& =\frac{1}{p_{\text {on }}(r, \tau, \eta)}\left\{\exp \left\{-\frac{1}{2} \boldsymbol{\Lambda}_{a}^{T} \boldsymbol{\Sigma}_{1} \boldsymbol{\Lambda}_{a}\right\}-\frac{\exp \left\{-\frac{1}{2} \boldsymbol{\Lambda}_{a}^{T} \boldsymbol{\Sigma}_{2} \boldsymbol{\Lambda}_{a}\right\}}{\eta \sqrt{\operatorname{Det}\left[\boldsymbol{B}+\boldsymbol{\sigma}_{\mathrm{M}}\right]}}\right\},
\end{aligned}
$$

with $\boldsymbol{\Sigma}_{1}=\boldsymbol{A}$ and $\boldsymbol{\Sigma}_{2}=\boldsymbol{A}-\boldsymbol{C}\left(\boldsymbol{B}+\boldsymbol{\sigma}_{\mathrm{M}}\right)^{-1} \boldsymbol{C}^{T}$. Note that the output state is no longer a Gaussian state, namely its characteristic function is no longer Gaussian: for this reason the IPS process is also referred to as de-Gaussification process [6].

In general, a Gaussian state described by the characteristic function [in Cartesian notation, namely $\left.\boldsymbol{\Lambda}=(\mathrm{x}, \mathrm{y})^{T}\right]$

$$
\chi(\boldsymbol{\Lambda})=\exp \left\{-\frac{1}{2} \boldsymbol{\Lambda}^{T} \boldsymbol{\sigma} \boldsymbol{\Lambda}\right\}
$$

with covariance matrix

$$
\boldsymbol{\sigma}=\left(\begin{array}{ll}
\mathrm{a} & \mathrm{c} \\
\mathrm{c} & \mathrm{b}
\end{array}\right)
$$

can be also written in the complex notation as follows:

$$
\chi(\lambda)=\exp \left\{-\mathcal{A}|\lambda|^{2}-\mathcal{B} \lambda^{2}-\mathcal{B}^{*} \lambda^{* 2}\right\},
$$

with

$$
\mathcal{A}=\frac{1}{2}(\mathrm{a}+\mathrm{b}), \quad \mathcal{B}=\frac{1}{4}(\mathrm{~b}-\mathrm{a}+2 i \mathrm{c}),
$$

where we introduced the complex number $\lambda=\frac{1}{\sqrt{2}}(x+i y)$. In this way, the characteristic function (17) can be written as follows:

$\chi_{r, \tau, \eta}^{\text {(out) }}(\lambda)=\frac{\exp \left\{-\mathcal{A}_{1}|\lambda|^{2}-\mathcal{B}_{1} \lambda^{2}-\mathcal{B}_{1}^{*} \lambda^{* 2}\right\}}{p_{\text {on }}(r, \tau, \eta)}-\frac{\exp \left\{-\mathcal{A}_{2}|\lambda|^{2}-\mathcal{B}_{2} \lambda^{2}-\mathcal{B}_{2}^{*} \lambda^{* 2}\right\}}{p_{\text {on }}(r, \tau, \eta) \eta \sqrt{\operatorname{Det}\left[\boldsymbol{B}+\boldsymbol{\sigma}_{\mathrm{M}}\right]}}$,

where $\mathcal{A}_{k}$ and $\mathcal{B}_{k}$ are refers to the covariance matrix $\Sigma_{k}, k=1,2$ respectively. Finally, using the definition

$$
W[\varrho](\alpha)=\frac{1}{\pi^{2}} \int_{\mathbb{C}} d^{2} \lambda \chi[\varrho](\lambda) \exp \left\{\lambda^{*} \alpha-\alpha^{*} \lambda\right\},
$$

which relates the Wigner function $W[\varrho](\alpha)$ of a state $\varrho$ to its characteristic function $\chi[\varrho](\lambda)$, one can obtain the Wigner function $W_{r, \tau, \eta}^{(\text {out })}(\alpha) \equiv W\left[\varrho_{r, \tau, \eta}^{\text {(out })}\right](\alpha)$. As for the characteristic function, to pass from the complex, $W[\varrho](\alpha)$, to the Cartesian notation, 

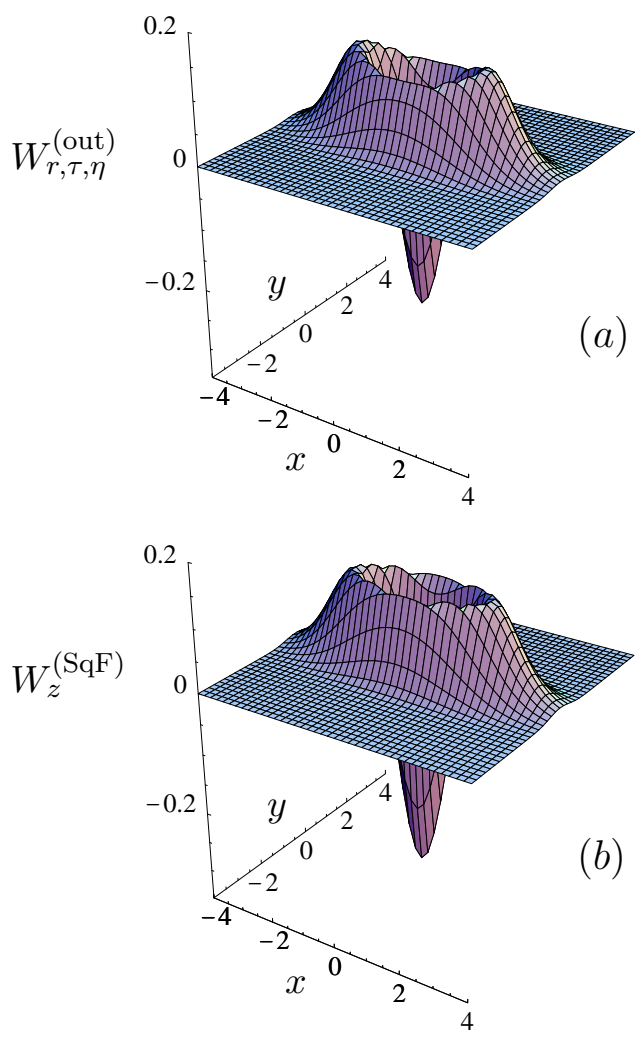

Figure 2. (a) Plot of the Wigner function $W_{r, \tau, \eta}^{(\text {out })}(x, y)$ with $r=0.5, \tau=0.90$, and $\eta=0.80$; (b) plot of the Wigner function $W_{z}^{(\mathrm{SqF})}(x, y)$ of the state $S(z)|1\rangle$ with squeezing parameter $z=0.5$.

$W[\varrho](x, y)$, one should put $\alpha=\frac{1}{\sqrt{2}}(x+i y)$ [7]. In figure 2 (a) we report $W_{r, \tau, \eta}^{(\text {out }}(x, y)$ for fixed $r, \tau$, and $\eta$ : as it is apparent from the plot the Wigner function is not Gaussian, and may assume negative values [6]. In Section 4 we will investigate this effect by analyzing the nonclassicality of the conditioned state. In figure2 (b) we show the Wigner function $\chi_{z}^{(\mathrm{SgF})}(x, y)$ associated to the squeezed Fock state $\varrho_{z}^{(\mathrm{SgF})}=S(z)|1\rangle\langle 1| S^{\dagger}(z)$, whose characteristic function $\chi_{z}^{(\mathrm{SgF})}(\lambda) \equiv \chi\left[\varrho_{z}^{(\mathrm{SqF})}\right](\lambda)$ reads (we assume $z$ as real)

$\chi_{z}^{(\mathrm{SqF})}(\lambda)=\left[1-2\left(\mathcal{A}_{0}|\lambda|^{2}+\mathcal{B}_{0} \lambda^{2}+\mathcal{B}_{0}^{*} \lambda^{* 2}\right)\right] \exp \left\{-\mathcal{A}_{0}|\lambda|^{2}-\mathcal{B}_{0} \lambda^{2}-\mathcal{B}_{0}^{*} \lambda^{* 2}\right\}$,

with $\mathcal{A}_{0}=2\left(\cosh ^{2} z+\sinh ^{2} z\right)$ and $\mathcal{B}_{0}=-2 \cosh z \sinh z$. Since the Wigner functions of the IPS squeezed vacuum and of the squeezed number state are quite similar, one can think of using the IPS process to produce the state $\varrho_{r}^{(\mathrm{SgF})}$; motivated by this consideration, in the next section we will analyses the fidelity between this states. Figure 3 shows $W_{r, \tau, \eta}^{(\text {out })}(x, y)$ with fixed $r$ and $\eta$ and different values of the IPS transmissivity $\tau$; the plots on the right of the same figure compare the $W_{r, \tau \eta}^{(\text {out })}(0, y)$ (solid lines) with $W_{r}^{(\mathrm{SgF})}(0, y)$ (dashed line). Finally, the effect of the quantum efficiency $\eta$ on the output state is shown in figure 4, where we plot as reference the value of the Wigner function $W_{r, \tau, \eta}^{(\text {out })}$ at the center of the complex plane as a function of the transmissivity $\tau$ and different values of $\eta$ : we can see that the main effect on the output state is due to $\tau$. 
Squeezed Fock state by inconclusive photon subtraction

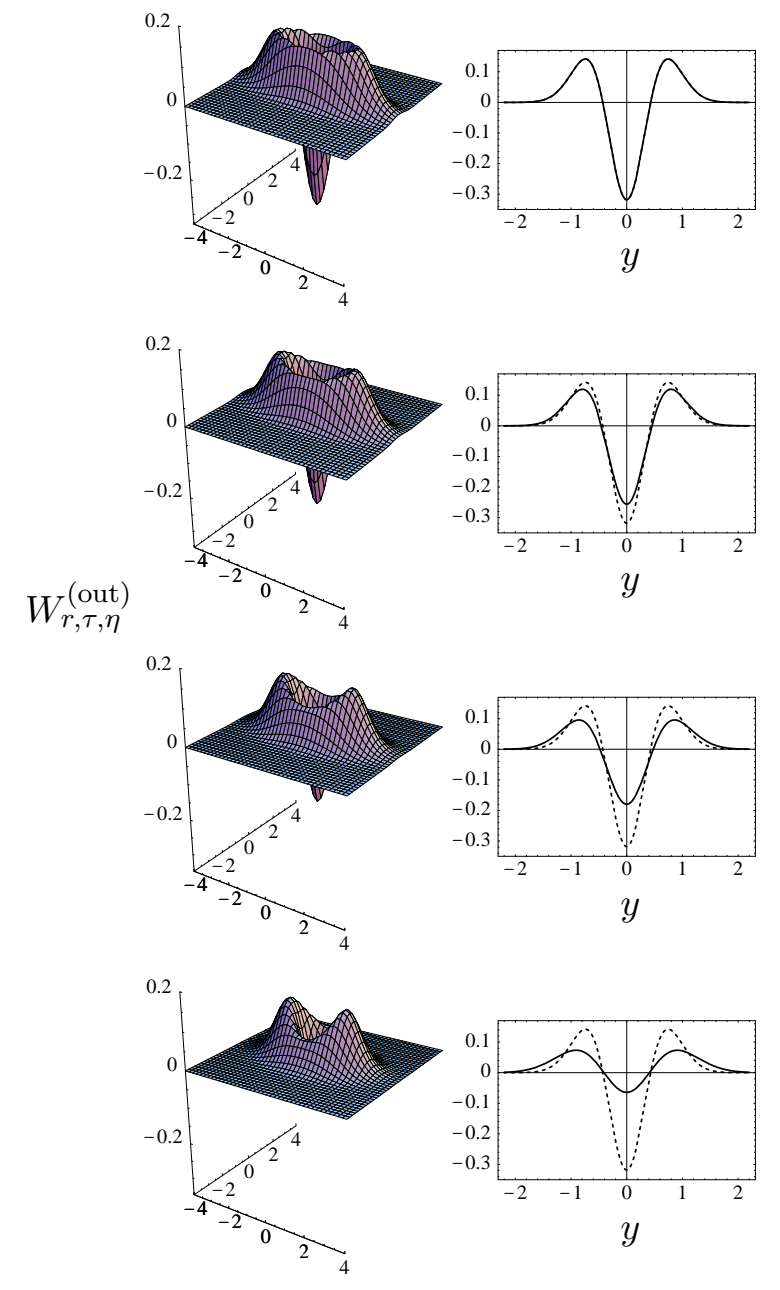

Figure 3. Plots of the Wigner function $W_{r, \tau, \eta}^{(\text {out })}(x, y)$ with $r=0.5, \eta=0.80$ and different values of the BS transmissivity $\tau$ : from top to bottom $\tau=0.99,0.9,0.75$, and 0.50. The solid lines of the plots on the right refer to $W_{r, \tau \eta}^{(\text {out })}(0, y)$ whereas the dashed lines are $W_{z}^{(\mathrm{SqF})}(0, y)$ of the state $S(z)|1\rangle$ with squeezing parameter $z=0.5$. Note that when $\tau=0.99$ the two lines overlap. $y$ is the squeezed coordinate.

\section{Fidelity and purity}

The fidelity between the pure state $\varrho_{z}^{(\mathrm{SqF})}$ and the IPS state $\varrho_{r, \tau, \eta}^{(\text {out })}$ is defined as follows:

$$
\begin{aligned}
F_{\tau, \eta}(z, r) & =\operatorname{Tr}\left[\varrho_{z}^{(\mathrm{SqF})} \varrho_{r, \tau, \eta}^{(\mathrm{out})}\right] \\
& =\frac{1}{2 \pi} \int_{\mathbb{R}^{2}} d^{2} \boldsymbol{\Lambda} \chi_{z}^{(\mathrm{SqF})}(\boldsymbol{\Lambda}) \chi_{r, \tau, \eta}^{(\mathrm{out})}(-\boldsymbol{\Lambda}), \\
& =\frac{1}{p_{\mathrm{on}}(r, \tau, \eta)}\left\{\mathcal{F}_{1}-\frac{\mathcal{F}_{2}}{\eta \sqrt{\operatorname{Det}\left[\boldsymbol{B}+\boldsymbol{\sigma}_{\mathrm{M}}\right]}},\right\}
\end{aligned}
$$

where

$$
\mathcal{F}_{k}=\frac{\mathcal{A}_{k}^{2}-\mathcal{A}_{0}^{2}-4\left(\mathcal{B}_{k}^{2}-\mathcal{B}_{0}^{2}\right)}{\left[\left(\mathcal{A}_{0}+\mathcal{A}_{k}\right)^{2}-4\left(\mathcal{B}_{0}+\mathcal{B}_{k}\right)^{2}\right]^{3 / 2}}
$$



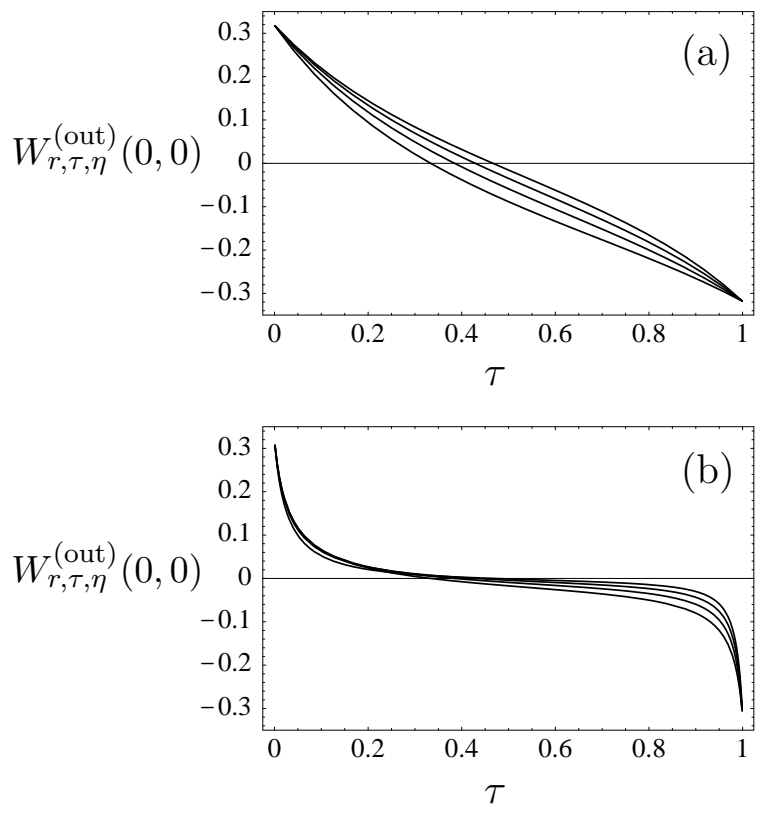

Figure 4. Plots of $W_{r, \tau, \eta}^{\text {(out) }}(0,0)$ with (a) $r=0.5$ and (b) $r=2.0$ as a function of $\tau$ and different values of $\eta$ : from bottom to top $\eta=1.0,0.75,0.50$, and 0.25 . The value of the function is mainly affected by $\tau$.

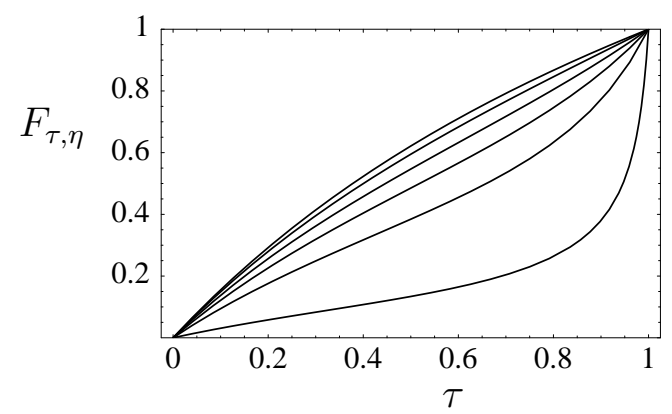

Figure 5. Plot of the fidelity $F_{\tau, \eta}(r)$ with $\eta=0.80$ as a function of the IPS transmissivity $\tau$ for different values of $r$ : from top to bottom $r=0.1,0.3,0.5,0.7,1.0$, and 2.0.

and $\mathcal{A}_{h}$ and $\mathcal{B}_{h}, h=0,1,2$, have been introduced in equations (24) and (22), respectively. The analytic expression of $F_{\tau, \eta}(z, r)$ is quite cumbersome, but, on the other hand, we can draw some interesting consideration by addressing its expansion at the first order in the transmissivity $\tau$ when $\tau \rightarrow 1$ and $\eta=1$, namely

$$
\begin{aligned}
F_{\tau, 1}(z, r)= & \frac{1}{\cosh ^{3}(r-z)} \\
& -\left[\frac{9 \cosh (r+z)-3 \cosh (3 r-z)}{8 \cosh (r-z)}-\frac{1}{4}\right](1-\tau)+o\left[(1-\tau)^{2}\right] .
\end{aligned}
$$

In fact, from the expansion (29) we conclude that the maximum of the fidelity is achieved when $z=r$. 


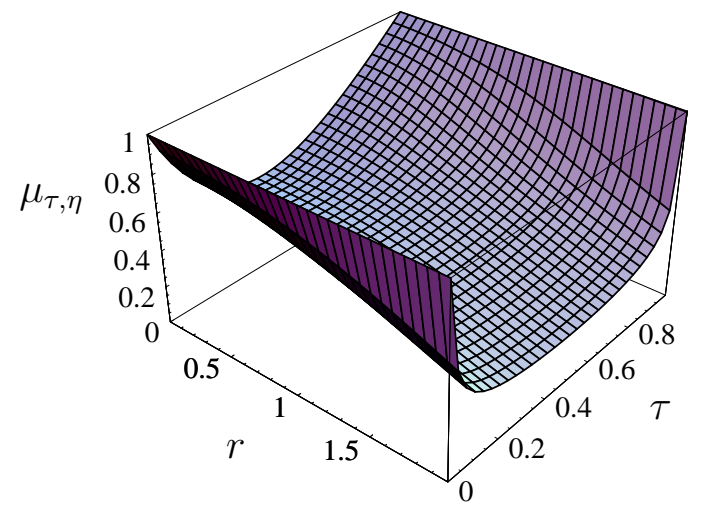

Figure 6. Plot of the purity $\mu_{\tau, \eta}(r)$ of the state $\varrho_{r, \tau, \eta}^{\text {(out) }}$. We set $\eta=0.80$.

In figure 5 we plot $F_{\tau, \eta}(r) \equiv F_{\tau, \eta}(r, r)$ as a function of the IPS transmissivity and for different values of $r$. We can see that $F_{\tau, \eta}$ reaches it maximum when the IPS transmissivity approaches 1 , namely in the single-photon subtraction limit [3]. Moreover, when the squeezing parameter $r$ increases the fidelity decreases: this is due to the increasing (unknown) number of subtracted photons which reduces the purity of the IPS state itself. In figure 6 we plot the purity $\mu_{\tau, \eta}(r)$ of the IPS squeezed vacuum $\varrho_{r, \tau, \eta}^{\text {(out) }}$, defined as follows [8]:

$$
\begin{aligned}
& \mu_{\tau, \eta}(r)=\operatorname{Tr}\left[\left(\varrho_{r, \tau, \eta}^{\text {(out })}\right)^{2}\right]=\pi \int_{\mathbb{C}} d^{2} \alpha\left[W_{r, \tau, \eta}^{\text {out }}(\alpha)\right]^{2} \\
&=\frac{1}{2 p_{\text {on }}(r, \tau, \eta)}\left\{\frac{1}{\sqrt{\mathcal{A}_{1}^{2}-4 \mathcal{B}_{1}^{2}}+\frac{1}{\eta^{2} \operatorname{Det}\left[\boldsymbol{B}+\boldsymbol{\sigma}_{\mathrm{M}}\right] \sqrt{\mathcal{A}_{1}^{2}-4 \mathcal{B}_{1}^{2}}}}\right. \\
&\left.-\frac{4}{\eta \sqrt{\operatorname{Det}\left[\boldsymbol{B}+\boldsymbol{\sigma}_{\mathrm{M}}\right]} \sqrt{\left(\mathcal{A}_{1}+\mathcal{A}_{2}\right)^{2}-4\left(\mathcal{B}_{1}+\mathcal{B}_{2}\right)^{2}}}\right\} .
\end{aligned}
$$

\section{Nonclassicality of the IPS squeezed vacuum state}

As a measure of nonclassicality of the IPS state $\varrho_{r, \tau, \eta}^{(\text {out })}$ we consider the nonclassical depth 9

$$
\mathcal{T}=\frac{1-\bar{s}}{2}
$$

$\bar{s}$ being the maximum $s$ for which the generalized quasi-probability function

$$
W_{s}(\alpha)=\frac{1}{\pi} \int_{\mathbb{C}} d^{2} \lambda \chi(\lambda) \exp \left\{\frac{1}{2} s+\lambda^{*} \alpha-\alpha^{*} \lambda\right\}
$$

is a probability distribution, i.e. positive semidefinite and non singular. As a matter of fact, one has $\mathcal{T}=1$ for number states and $\mathcal{T}=0$ for coherent states. Moreover, the nonclassical depth can be interpreted as the minimum number of thermal photons which has to be added to a quantum state in order to erase all the quantum features of 
the state [7, 9]. In the case of $\varrho_{r, \tau, \eta}^{(\text {out })}$, we have [for the sake of simplicity we do not write explicitly the dependence on $r, \tau$ and $\eta$ in the symbol $\left.W_{s}^{(\text {out })}(\alpha)\right]$

$$
W_{s}^{(\text {out })}(\alpha)=\frac{1}{p_{\text {on }}(r, \tau, \eta)}\left\{\mathcal{G}_{1}(\alpha)-\frac{\mathcal{G}_{2}(\alpha)}{\eta \sqrt{\operatorname{Det}\left[\boldsymbol{B}+\boldsymbol{\sigma}_{\mathrm{M}}\right]}}\right\}
$$

where we defined

$$
\mathcal{G}_{k}(\alpha)=\frac{2 \exp \left\{-\frac{2\left(2 \mathcal{A}_{k}-s\right)|\alpha|^{2}+4 \mathcal{B}_{k}^{*} \alpha^{2}+4 \mathcal{B}_{k} \alpha^{* 2}}{\left(2 \mathcal{A}_{k}-s\right)^{2}-16\left|\mathcal{B}_{k}\right|^{2}}\right\}}{\pi \sqrt{\left(2 \mathcal{A}_{k}-s\right)^{2}-16\left|\mathcal{B}_{k}\right|^{2}}} .
$$

At first we note that in order to have $W_{s}^{(\text {out })}(\alpha)$ normalizable, equation the following condition should be satisfied

$$
s \leq 2 \mathcal{A}_{k} \quad(k=1,2) .
$$

Furthermore, since $W_{s}^{(\text {out })}(\alpha)$ is a difference between two Gaussian functions with the center in the origin of the complex plane, one can easily see that, in general, this function has a minimum in $\alpha=0$ and that this minimum can be negative. For this reason and thanks to other simple considerations about the symmetries of $W_{s}^{(\text {out })}(\alpha)$ with respect to the point $\alpha=0$, we can focus our attention in the origin of the complex plane, obtaining this further condition for the positivity:

$$
\mathcal{G}_{1}(0)-\frac{\mathcal{G}_{2}(0)}{\eta \sqrt{\operatorname{Det}\left[\boldsymbol{B}+\boldsymbol{\sigma}_{\mathrm{M}}\right]}} \geq 0,
$$

which, together with the conditions (36), brings to

$$
\bar{s}(\tau, \eta)=\frac{2-\eta-(4-\eta) \tau}{2-(1-\tau) \eta}
$$

and, then, to the following expression for the nonclassical depth:

$$
\mathcal{T}(\tau, \eta)=\frac{2 \tau}{2-(1-\tau) \eta}
$$

Since $\mathcal{T}(\tau, \eta) \geq 0$, the conditional state is nonclassical for any non-zero value of the IPS transmissivity and efficiency. Note that equation (39) depends only on $\tau$ and $\eta$, whereas it is independent on the squeezing parameter $r$. Notice, however, the nonclassical depth does not measure the extension of the negativity region, but only the presence of negative values. Therefore it is not surprising that equation (39) does not depend on $r$. We plot $\mathcal{T}(\tau, \eta)$ in figure [7. Since the usual Wigner function is obtained when $s=0$ in (33), from equation (38) we can see that $W_{r, \tau, \eta}^{(\text {out })}(\alpha)$ becomes semi-positive definite when $\tau=(2-\eta) /(4-\eta)$.

\section{Concluding remarks}

We have analyzed in details the state obtained subtracting photons from the squeezed vacuum by means of linear optics, namely using beam splitters and avalanche photodetectors. We referred to the whole photon-subtraction process as to inconclusive 


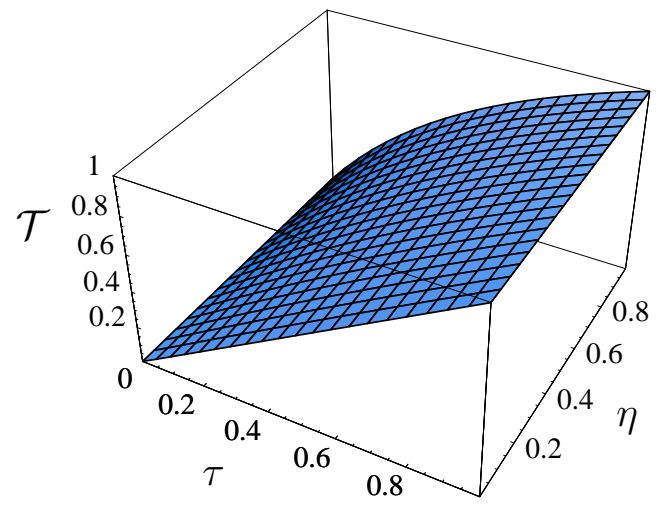

Figure 7. Plot of the nonclassical depth $\mathcal{T}(\tau, \eta)$ of the IPS squeezed vacuum state.

photon subtraction (IPS), since avalanche photodetectors are not able to resolve the number of detected photons. We found that the IPS conditional state obtained from a squeezed vacuum state is close to the squeezed Fock state $S(r)|1\rangle$ and approaches this target state when only one photon is subtracted, namely, using a high transmissivity beam splitter for the IPS. Moreover, when the transmissivity and the quantum efficiency are not unitary, the output state remains close to the target state, showing a high fidelity for a wide range of the parameters. The purity and the nonclassicality of the IPS squeezed vacuum state have been also considered: we found that the relevant parameter is the transmissivity $\tau$, while the IPS efficiency $\eta$ only slightly affects the output state. We conclude that IPS, which was recently experimentally implemented [6], can be effectively used to produce a nonclassical state such as the squeezed Fock state $S(r)|1\rangle$, whose generation would be, otherwise, quite challenging.

\section{References}

[1] T. Opatrný, G. Kurizki, and D.-G. Welsch, Phys. Rev. A 61, 032302 (2000).

[2] P. T. Cochrane, T. C. Ralph, and G. J. Milburn, Phys. Rev. A 65, 062306 (2002).

[3] M. G. A. Paris, Phys. Lett. A 289, 167 (2001); S. Olivares, M. G. A. Paris, and R. Bonifacio, Phys. Rev. A 67,032314 (2003).

[4] S. Olivares, and M. G. A. Paris, Phys. Rev. A 70, 032112 (2004).

[5] J. Eisert, D. E. Browne, S. Scheel, and M. B. Plenio, Ann. Phys. 311, 431 (2004); D. E. Browne, J. Eisert, S. Scheel, and M. B. Plenio, Phys. Rev. A 67, 062320 (2003).

[6] J. Wenger, R. Tualle-Bouri, and P. Grangier, Phys. Rev. Lett. 92, 153601 (2004).

[7] A. Ferraro, S. Olivares, and M. G. A. Paris, Gaussian States in Quantum Information (Bibliopolis, Napoli, 2005).

[8] M. G. A. Paris, F. Illuminati, A. Serafini, and S. De Siena, Phys. Rev. A 68, 012314 (2003); M. S. Kim, J. Lee, and W. J. Munro, Phys. Rev A 66, 030301 (2002).

[9] C. T. Lee, Phys. Rev. A 44, R2775 (1991). 